\title{
The use of the international GPS network as the global detector (GLOBDET) simultaneously observing sudden ionospheric disturbances
}

\author{
Edward L. Afraimovich, Eugene A. Kosogorov, and Ludmila A. Leonovich \\ Institute of Solar-Terrestrial Physics SD RAS, p. o. box 4026, Irkutsk 664033, Russia \\ (Received January 17, 2000; Revised September 2, 2000; Accepted September 5, 2000)
}

\begin{abstract}
We developed a new technology for global detection of ionospheric disturbances, on the basis of phase measurements of the total electron content (TEC) along the line-of-sight (LOS) between the receiver on the ground and transmitters on the GPS satellites using an international GPS networks. Temporal dependencies of TEC with the time resolution of $30 \mathrm{~s}$ are obtained for a set of spaced receivers of the GPS network simultaneously for the entire set of visible satellites. These series are subjected to filtering in the selected range of oscillation periods using known algorithms for spatio-temporal analysis of signals. An analysis is made of the possibilities of using the GLOBDET when detecting the ionospheric response of solar flares. In this case it is best to make the coherent summation of the filtered series of TEC. Because of a statistical independence of the background fluctuations, the signal/noise ratio, when the flare effect is detected, is increased due to a coherent processing by at least the $\sqrt{N}$ times, where $N$ is the number of LOS.
\end{abstract}

\section{Introduction}

The advent and evolution of a Global Positioning System, GPS, and also the creation on its basis of widely branched networks of GPS stations (at least 732 sites at August of 2000, the data from which are placed on the INTERNET) opened up a new era in remote ionospheric sensing (Klobuchar, 1997). Furthermore, there exist also powerful regional networks such as the Geographical Survey Institute network in Japan (Saito et al., 1998) consisting of up to 1000 receivers. Highprecision measurements of the group and phase delay along the line-of-sight (LOS) between the receiver on the ground and transmitters on the GPS system satellites covering the reception zone are made using two-frequency multichannel receivers of the GPS system at almost any point of the globe and at any time simultaneously at two coherently coupled frequencies $f_{1}=1575.42 \mathrm{MHz}$ and $f_{2}=1227.60 \mathrm{MHz}$.

The sensitivity of phase measurements in the GPS system is sufficient for detecting irregularities with an amplitude of up to $10^{-3}-10^{-4}$ of the diurnal total electron content (TEC) variation. This makes it possible to formulate the problem of detecting ionospheric disturbances from different sources of artificial and natural origins. Anthropogenic sources of ionospheric disturbances include nuclear events, chemical explosions, and rocket launches. Among natural sources are solar eclipses, solar flares, earthquakes, volcanoes, heavy thunderstorms, and auroral heating. Studies of these phenomena are of great importance both for a scientific understanding of their genesis and propagation in the atmosphere and as a tool for detecting such sources in remote regions.

Recently a number of authors (for example, Mannucci

Copy right (c) The Society of Geomagnetism and Earth, Planetary and Space Sciences (SGEPSS); The Seismological Society of Japan; The Volcanological Society of Japan; The Geodetic Society of Japan; The Japanese Society for Planetary Sciences. et al., 1998 and others) have developed a new technology for constructing global maps of absolute 'vertical' values of TEC using data from the GPS network (Global Ionospheric Maps-GIM technology). Coupled with the possibility of obtaining these maps in the standard IONEX format on the INTERNET, the GIM technology may be used for the global detection of the large-scale ionospheric disturbances (Ho et al., 1998), but with low time resolution of the maps. Ho et al. (1998) have TEC maps with time steps of $15 \mathrm{~min}$ at their disposal. Now two-hour resolution data are available on the INTERNET. At the same time, TEC measurements at each particular point of the GPS systems are made with 30-second intervals.

Thus the GIM technology cannot be used in detecting short-lasting processes with a duration of about 1-10 min, much less in detecting virtually simultaneous disturbances on a global scale. A most dramatic example of such disturbances is the ionospheric response to a solar flare (Mitra, 1974). Research has also been done into ionospheric irregularities on global-scales (Pi et al., 1997) or regional-scales (Aarons et al., 1997), based on estimating the rate of TEC change. However, the recordings of this parameter in the cited references were not used in detecting simultaneous disturbances such as solar flares.

The objective of this paper is to develop the concept of a global detector (GLOBDET) of "simultaneous" ionospheric disturbances on global-scale, based on phase measurements of the TEC in the ionosphere using the international network of two-frequency multichannel receivers of the GPS system with the time resolution of $30 \mathrm{~s}-$ Section 2. Section 3 discusses the possibilities of using the GLOBDET method in detecting ionospheric effects of solar flares. Basic characteristics of such a detector (sensitivity and time resolution) are examined in Section 4. The practical implementation of 
the method is illustrated by analyzing a global ionospheric response to a powerful solar flare of July 29, 1999 (Section 5).

\section{Using the Data from a Global GPS Network in the Context of the Ideology of a Phased Antenna Array}

Phased antenna arrays (PAA) are extensively used in radiolocation, sonar systems, and in processing seismic signals as systems featuring high sensitivity in signal detection and high spatial selectivity which is necessary for localizing signal sources. There exist quite varied schemes for hardware and software realization of PAA (Collin, 1985). In general case the treatment of signals imply multiplying a complex signal $\tilde{A}(t)_{i}$ of each of the $i$-spaced PAA elements by a complex factor $\tilde{K}_{i}$ with the subsequent coherent summation of the resulting products

$$
\tilde{S}=\sum_{i=1}^{N} \tilde{A}(t)_{i} \tilde{K}_{i}
$$

where $\tilde{S}$ is the result of the coherent summation; $i$ is the PAA element number; and $i=1,2, \ldots, N$.

By specifying different values of $\tilde{K}_{i}$, which in each particular case depend on the conditions of the problem being solved, it is possible to specify parameters determining the sensitivity and selectivity of PAA.

We suggest the concept of a global detector (GLOBDET) of ionospheric disturbances of natural and technogenic origins, based on processing the GPS data in the PAA configuration. This concept implies that time dependencies of TEC are obtained for a set of spaced receivers of the GPS network simultaneously for all GPS satellites "visible" during a given time interval. These series are subjected to filtering over a selected range of oscillation periods in order to eliminate slow variations caused by the orbital motion of the satellites and by the diurnal variation of TEC in the ionosphere. Next, these series are processed in the nonequidistant PAA configuration using them in (1) or in other algorithms of PAA as complex signals $\tilde{A}(t)_{i}$. It is also assumed that equivalent partial antennas of such an array are located at subionospheric points. The coordinates of these points are determined for the selected height $h_{s}$ by means of standard (in the GPS system) calculations of the azimuth $\alpha$ and elevation $\theta$ of the LOS between the receiver and the satellite. $\alpha$ and $\theta$ are reckoned from the northward direction and from the ground, respectively.

The GPS technology provides the means of estimating TEC variations on the basis of phase measurements of TEC $I$ in each of the spaced two-frequency GPS receivers using the formula (Afraimovich et al., 1998):

$$
I=\frac{1}{40.308} \frac{f_{1}^{2} f_{2}^{2}}{f_{1}^{2}-f_{2}^{2}}\left[\left(L_{1} \lambda_{1}-L_{2} \lambda_{2}\right)+\text { const }+n L\right]
$$

where $L_{1} \lambda_{1}$ and $L_{2} \lambda_{2}$ are phase path increments of the radio signal, caused by the phase delay in the ionosphere (m); $L_{1}$, $L_{2}$ is the number of full phase rotations, and $\lambda_{1}$, and $\lambda_{2}$, are the wavelengths $(\mathrm{m})$ for the frequencies $f_{1}$ and $f_{2}$, respectively; const is some unknown initial phase path (m); and $n L$ is the error in determination of the phase path $(\mathrm{m})$.

The TEC unit, TECU, which is equal to $10^{16} \mathrm{~m}^{-2}$ and is commonly accepted in the literature, will be used throughout the text.

\section{Using the GLOBDET Technology in Detecting the Ionospheric Response to Solar Flares}

The enhancement of X-ray and ultraviolet radiation intensity that is observed during chromospheric flares on the Sun immediately causes an increase in electron density in the ionosphere. These density variations are different for different altitudes and are collectively called Sudden Ionospheric Disturbances (SID). SID observations provide a key means for ground-based detection of solar flares along with optical observations of flares and solar radio burst observations (Mitra, 1974).

SID data for the $F$-region acquired by different radio probing methods were used repeatedly to estimate time variations in the X-ray and extreme ultraviolet (EUV) spectral regions and in relative measurements of fluxes in different wavelength ranges (Mitra, 1974). The main body of SID data for the Earth's upper atmosphere was obtained in earlier detections of Sudden Frequency Deviations (SFD) of the $F$ region-reflected radio signal in the HF range (Davies, 1969; Liu et al., 1996).

SFD are caused by an almost time-coincident increase in $E$ - and $F$-region electron densities at over $100 \mathrm{~km}$ altitudes covering an area with the size comparable to or exceding that of the region monitored by the system of HF radio paths. A limitation of this method is the uncertainty in the spatial and altitude localization of the UV flux effect, the inadequate number of paths, and the need to use special-purpose equipment.

A further, highly informative, technique is the method of incoherent scatter (IS)_-Thome and Wagner (1971). However, the practical implementation of the IS method requires very sophisticated, expensive equipment. An added limitation is inadequate time resolution. Since the relaxation time of electron density in the $E$ - and $F 1$-regions is also less than 5-10 min, most IS measurements lack time resolution needed for the study of inospheric effects of flares.

The effect of solar flares on the ionospheric $F$-region is also manifested as a Sudden Increase of TEC (SITEC) which was measured previously using continuously operating radio beacons on geostationary satellites (Mendillo et al., 1974). A serious limitation of methods based on analyzing VHF signals from geostationary satellites is their small and ever increasing with the time number and the nonuniform distribution in longitude.

Consequently, none of the above-mentioned existing methods can serve as an effective basis for the radio detection system to provide a continuous, global SID monitoring with adequate space-time resolution. Furthermore, the creation of these facilities requires developing special-purpose equipment, including powerful radio transmitters contaminating the radio environment. It is also significant that when using the existing methods, the inadequate spatial aperture gives no way of deducing the possible spatial inhomogeneity of the X-ray and EUV flux.

According to the above concept, a global GPS network can be successfully used as a global detector of the ionospheric response to solar flares. A physical groundwork for the method is formed by the effect of fast change in electron density in the Earth's ionosphere at the time of a flare simultaneously on the entire sunlit surface. 
Essentially, the method implies using appropriate filtering and a coherent processing of TEC variations in the ionosphere simultaneously for the entire set of "visible" (during a given time interval) GPS satellites (as many as 5-10 satellites) at all global GPS network stations used in the analysis. In detecting solar flares, the ionospheric response is virtually simultaneous for all stations on the dayside of the globe within the time resolution range of the GPS receivers (from $30 \mathrm{~s}$ to $0.1 \mathrm{~s}$ ). Therefore, the coherent addition of individual realizations $\tilde{A}(t)_{i}$ does not require a partial phase shift (the complex part of the term $\tilde{K}_{i}$ is zero), and the entire procedure reduces to a simple addition

$$
\tilde{S}=\sum_{i=1}^{N} \tilde{A}(t)_{i} K_{i}
$$

where $\tilde{A}(t)_{i}$ represents filtered TEC variations, and $K_{i}$ is the amplitude weighting factor determined by the geometry of the $i$-beam (LOS) to the satellite. To a first approximation, this factor is (Klobuchar, 1986).

$$
K_{i}=\cos \left[\arcsin \left(\frac{R_{z}}{R_{z}+h_{s}} \cos \theta_{i}\right)\right]
$$

where $R_{z}$ is the Earth's radius; $h_{s}=300 \mathrm{~km}$ is the height for the subionospheric point where the conversion is taken place. The $h_{s}$ can be chosen at other altitudes, such as 350 $\mathrm{km}, 400 \mathrm{~km}$, or $450 \mathrm{~km}$.

Reconstructing the absolute value of the ionospheric response to the solar flare requires a more accurate (than used in this paper) conversion of the "oblique" TEC value to a "vertical" one, especially at low values of elevations of the beam to the satellite. To do this, it is necessary not only to eliminate, for this beam to the satellite, the ambiguity of the determination of the absolute TEC value which arises when only phase measurements are used in the GPS system. The response can only be estimated reliable, with the inclusion the spatially inhomogeneous ionosphere, by using all beams to the satellite, and by applying adequate methods of spatial interpolation. This problem is considered in a series of publications (for example, Mannucci et al., 1998) and is beyond the scope of this paper.

\section{Characteristics of a Global Detector}

It is impossible to give a detailed account of the characteristics of the proposed global detector for reasons of space. This is beyond the scope of this study and will be reported elsewhere. We limit our attention to discussing only some general parameters of such a detector such as sensitivity and time resolution.

The detection sensitivity is determined by the ability to detect typical signals of the ionospheric response to a solar flare (leading edge duration, period, form, length) at the level of TEC background fluctuations. Ionospheric irregularities are characterized by a power spectrum, so that background fluctuations will always be distinguished in the frequency range of interest. However, background fluctuations are not correlated in the case of beams to the satellite spaced by an amount exceeding the typical irregularity size.

With a typical length of X-ray bursts and EUV emission of solar flares of about 5-10 min, the corresponding ionization irregularity size does normally not exceed $30-50 \mathrm{~km}$; hence the condition of a statistical independence of TEC fluctuations at spaced beams is almost always satisfied. Therefore, coherent summation of responses to a flare on a set of beams spaced thoughout the dayside of the globe permits the solar flare effect to be detected even when the response amplitude on partial beams is markedly smaller than the noise level (background fluctuations). The proposed procedure of coherent accumulation is essentially equivalent to the operation of coincidence schemes which are extensively used in X-ray and gamma-ray telescopes.

If the SID response and background fluctuations, respectively, are considered to be the signal and noise, then as a consequence of a statistical independence of background fluctuations the signal/noise ratio when detecting the flare effect is increased through a coherent processing by at least a factor of $\sqrt{N}$, where $N$ is the number of LOS.

The solar flare of July 29, 1999 was used to illustrate the performance of the proposed method. Figure 1 presents the geometry of a global GPS array used in this paper to analyze the effects of this flare (105 stations). The coordinates of the stations are not given here for reasons of space.

As is evident from Fig. 1, the set of stations which we chose out of the global GPS network available to us, covers rather densely North America and Europe, but provides much worse coverage of the Asian part of the territory used in the analysis. The number of GPS stations in the Pacific and Atlantic regions is even fewer. However, coverage of the ter-

\section{July $1999 ; 19: 30$ UT}

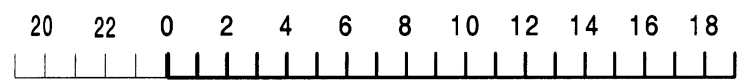

Latitude, $\mathrm{N}$

LT

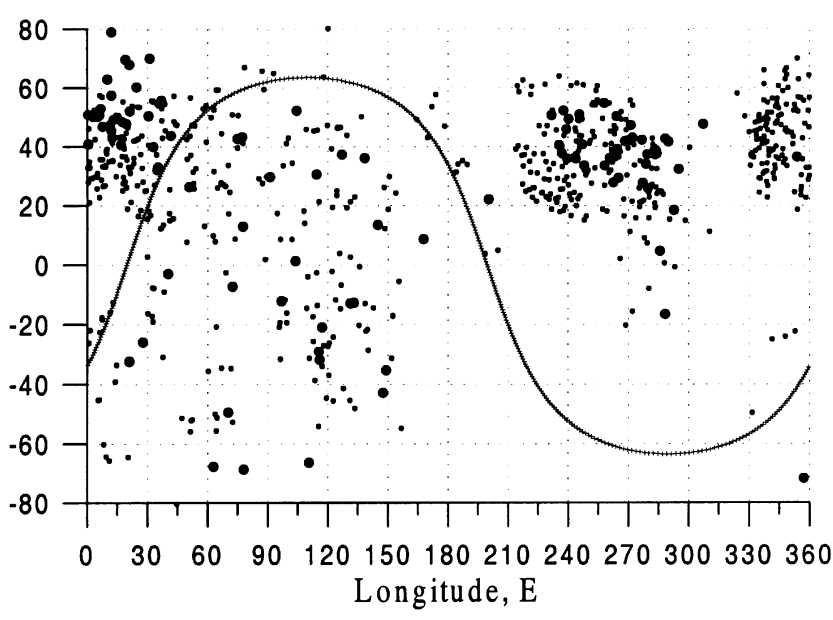

Fig. 1. Geometry of the GPS array used in this paper when analyzing the effects of the July 29, 1999 solar flare (105 stations). Heavy dots correspond to the location of the GPS stations. The upper scale indicates the local time, LT, corresponding to 19:30 UT, a maximum increase in X-ray emission intensity of the flare. Dots show the coordinates of subionospheric points for the height $h_{s}=300 \mathrm{~km}$ for all satellites seen at 19:30 UT for each of the GPS stations. A total number of beams (and subionospheric points) is 622. Dashes designate the terminator line for 19:30 UT. 
ritory with partial beams to the satellite for the limitation on elevations $\theta_{s}>10^{\circ}$, which we have selected, is substantially wider. Dots in Fig. 1 mark the coordinates of subinospheric points for the height $h_{s}=300 \mathrm{~km}$ for all visible satellites at 19:30 UT for each GPS station. A total number of beams (and subionospheric points) used in this paper to analyze the July 29, 1999 flare is 622.

Such coverage of the terrestrial surface makes it possible to solve the problem of detecting time-coincident events with spatial resolution (coherent accumulation) two orders of magnitude higher, as a minimum, than could be achieved in SFD detection on oblique HF paths (Davies, 1969; Liu et al., 1996). For simultaneous events in the western hemisphere, the corresponding today's number of stations and beams can be as many as 400 and $2000-3000$, respectively.

It should be noted that because of the relatively low satellite orbit inclinations, the GPS network (and to a lesser degree GLONASS) provides poor coverage of the Earth's surface near the poles. However, TEC measurements in the polar regions are ineffective with respect to the detection of the ionospheric response to a solar flare because the amplitude of background fluctuations in this case is much higher when compared with the mid-latitude ionosphere. This is particularly true of geomagnetic disturbance periods. For the same reason, equatorial stations should also be excluded from a coherent processing.

If the Earth's ionosphere is regarded as the filling of some global detector of X-ray and EUV emissions, then it is possible to estimate a huge volume $V_{\text {det }}$ of the potential sensitivity region of such a detector. This volume is equal to one-half the difference of the volumes of spheres with the radii $R_{z}+H_{\max }$ and $R_{z}+H_{\min }$

$$
V_{\text {det }}=2 \pi\left[\left(R_{z}+H_{\max }\right)^{3}-\left(R_{z}+H_{\min }\right)^{3}\right] / 3
$$

where $H_{\min }$ and $H_{\max }$ are the upper and lower boundaries of the absorbing layer for a given part of the flare emission spectrum; and $R_{z}$ is the Earth's radius. For the EUV range, $H_{\min }=100 \mathrm{~km}$, and $H_{\max }=200 \mathrm{~km}$ (Mitra, 1974), which gives the volume $V_{\text {det }}$ of order $2.65 \times 10^{19} \mathrm{~m}^{3}$. For the Xray part of the spectrum, $H_{\min }=60 \mathrm{~km}$, and $H_{\max }=80$ $\mathrm{km}$ (Mitra, 1974), and the corresponding volume is $0.523 \times$ $10^{19} \mathrm{~m}^{3}$.

The actual sensitivity is in fact determined by the number of beams penetrating the region of potential sensitivity. Furthermore, solar flare-induced TEC perturbations constitute but a small part of TEC including the height range up to $1000 \mathrm{~km}$. There are methods to estimate the height in the ionosphere, but they do not come within the province of this paper.

On the one hand, GLOBDET time resolution is limited by technical capabilities of the GPS system. Essentially, data with a time resolution of about $30 \mathrm{~s}$ are currently available on the INTERNET, which is insufficient for a detailed analysis of the fine structure of the SID time dependence. Yet this limitations seems to be transient since current multichannel two-frequency GPS receivers can operate with a time resolution of up to $0.1 \mathrm{~s}$.

On the other hand, time resolution is determined by time constants of ionization and recombination processes in the ionosphere at a given height (Mitra, 1974); these parameters can be taken into acount when processing the data.

\section{Ionospheric Response to the Solar Flare of July 29, 1999}

A powerful impulsive flare of July 29, 1999 was chosen to illustrate the practical implementation of the proposed method. Figure 2d (and Fig. 3d) shows the time dependence of the X-ray emission intensity $R_{X}(t)$ of this flare as measured by the X-telescope BATSE on satellite CGRO in $25-50 \mathrm{keV}$ range. The envelope of the dependence $R_{X}(t)$ represents a bell-shaped pulse of about 3-min duration with a maximum corresponding to 19:34 UT, or to about 12:00 LT in the U.S. West (Fig. 1). This time interval is characterized by a low level of geomagnetic disturbance (within -10 nT), which simplified greatly the SID detection problem.

We now describe briefly the sequence of GPS data processing procedures. Primary data include series of "oblique" values of TEC $I(t)$, as well as the corresponding series of elevations $\theta_{s}(t)$ and azimuths $\alpha_{s}(t)$ along LOS to the satellite calculated using our developed CONVTEC program which converts the GPS system standard RINEX-files on the INTERNET (Gurtner, 1993). The determination of SID characteristics involves selecting continuous series of $I(t)$ measurements of at least a one-hour interval in length, which includes the time of the flare. Series of elevations $\theta_{s}(t)$ and azimuths $\alpha_{s}(t)$ of the beam to the satellite are used to determine the coordinates of subionospheric points. In the case

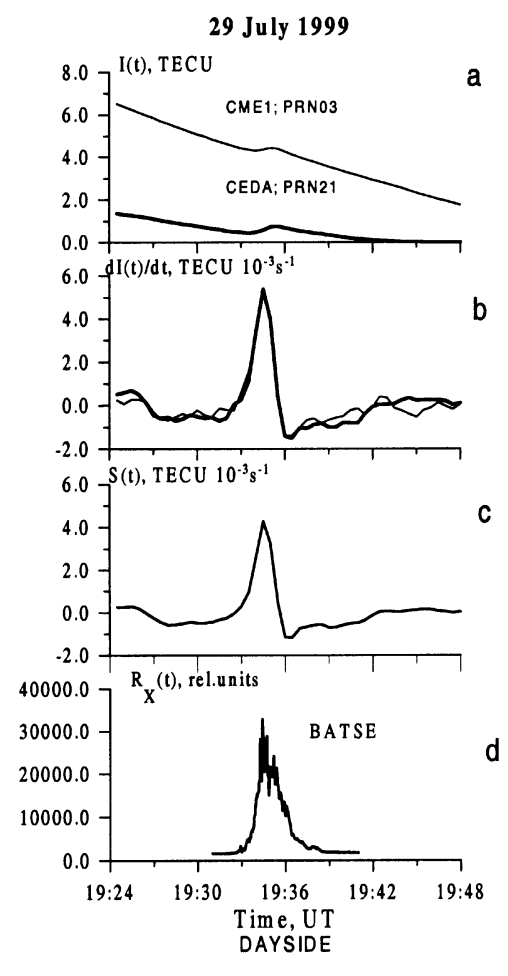

Fig. 2. Time dependences of TEC on the dayside $I(t)$ on July 29, 1999-a., and variations of the time derivative $d I(t) / d t$ with the linear trend removed and a smothing with the 5-min time window-b. for stations CME1 (PRN03-thick line) and CEDA (PRN21-thin line). The normalized coherent sum of variations of the TEC time derivative $S(t)$ for all LOS-c. For comparison, line in panel d. shows X-ray emission intensity variations $R_{X}(t)$ of the July 29, 1999 flare as deduced from the X-telescope BATSE on satellite CGRO data in $25-50 \mathrm{keV}$ range. 


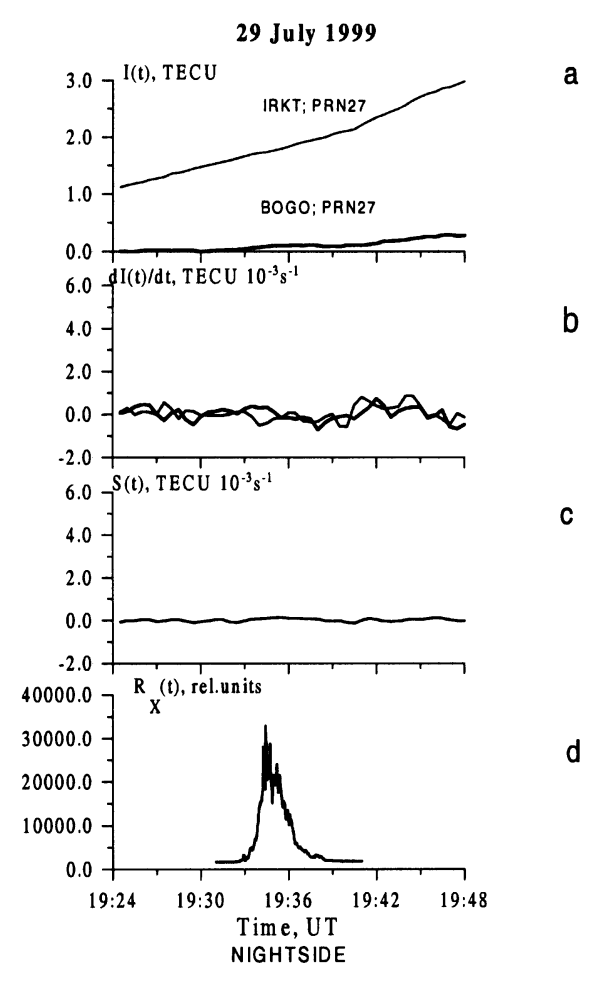

Fig. 3. Same as in Fig. 2, but for the nightside. Selected data for sites IRKT (PRN27) and BOGO (PRN27) are presented in panels a and b.

under consideration, all results were obtained for elevations $\theta_{s}(t)$ larger than $10^{\circ}$.

Figure 2a presents typical time dependencies of an "vertical" TEC $I(t)$ for the PRN03 satellite at the CME1 station $\left(40.4^{\circ} \mathrm{N} ; 235.6^{\circ} \mathrm{E}\right)$ on July 29,1999 (thick line) and for PRN21 at the CEDA station $\left(40.7^{\circ} \mathrm{N} ; 247.1^{\circ} \mathrm{E}\right.$ - thin line $)$. It is apparent from Fig. 2a that in the presence of slow TEC variations, the SID-induced short-lasting sudden increase in TEC is clearly distinguished in the form of a "step" as large as $0.4 T E C U$.

For the same series, similar lines in panel b show variations of the time derivative of TEC $d I(t) / d t$ with the linear trend removed and with a smoothing with the 5-min time window. The $d I(t) / d t$ variations for different beams are well correlated over the time interval from 19:30 to 19:39 UT. The TEC time derivative is invoked because it reflects electron density variations which are proportional to the Xray or EUV flux (Mitra, 1974; Liu et al., 1996). The time interval $d t$ for $d I / d t$ computation is $30 \mathrm{~s}$.

The coherent summation of $d I(t) / d t_{i}$ realizations was made by the formula (3). The (normalized to $N$ ) result of a coherent summation of the $S(t)$-series for all beams and GPS stations located mainly on the dayside is presented in panel c. A comparison of the resulting coherent sum of $S(t)$ with the time dependence $d I(t) / d t$ for individual beams presented in panels $b$ confirms the effect of a substantial increase of the signal/noise ratio caused by a coherent processing.

It is interesting to compare, for the same time interval, the data on individual beams and results from a coherent summation for the dayside and nightside. Figure 3 a presents typical time dependencies of an "oblique" TEC $I(t)$ for the
PRN27 satellite at the IRKT station $\left(52.2^{\circ} \mathrm{N} ; 104.3^{\circ} \mathrm{E}\right.$ - thick line) and for PRN27 at the BOGO station $\left(52.5^{\circ} \mathrm{N} ; 20.0^{\circ} \mathrm{E}-\right.$ thin line). Using the $I(t)$ data it is impossible to identify any SID-induced short-lasting sudden increase in TEC. This is also true for the time derivatives $d I(t) / d t$ plotted in panels $b$. As a result, the r.m.s. of the coherent sum of $S(t)$ in panel c for the nightside is of the same order of magnitude as that of background fluctuations outside the SID response interval on the dayside, which is an order of magnitude (as a minimum) smaller than the SID response amplitude (Fig. 2c).

A comparison of the coherent sum of $S(t)$ with the time dependence of the X-ray emission intensity $R_{X}(t)$ of the July 29, 1999 flare, based on the data from the X-telescope BATSE on satellite CGRO data in 25-50 keV range, shows their high correlation and an almost total coincidence of the form of $S(t)$ with the $R_{X}(t)$ pulse envelope at 19:33-19:38 UT time interval (Fig. 2d). The same time dependence of $\mathrm{X}$-rays is shown in Fig. 3d in order to demonstrate the lack of TEC response during the solar flare on the nightside.

Unfortunately, the lack of data on UV emission of the flares under investigation, the most probable ionizing factor at ionospheric heights above $100 \mathrm{~km}$, gives no way of making absolute estimates of the TEC increment and comparing them with measured values. It is pointed out in Mitra (1974) that although UV emission is essentially responsible for SID in the $F$-region, TEC variations are also correlated quite well with X-ray flares. This is also confirmed by simultaneous measurements of X-ray and EUV flare emission characteristics by the Solar Maximum Mission satellite (Vanderveen et al., 1988).

\section{Conclusions}

This paper has offered a brief outline of the concept of a global detector (GLOBDET) of ionospheric disturbances, based on phase measurements of a TEC in the ionosphere which were made using the international network of twofrequency multichannel GPS receivers. We have discussed the possibilities of using GLOBDET method in detecting ionospheric effects of solar flares. A case study of a global ionospheric response to a powerful solar flare of July 29, 1999 illustrates the new experimental potential.

For powerful solar flares like the one examined in this report, it is not necessary to invoke a coherent summation, and the SID response can be investigated for each beam. This opens the way to a detailed study of the SID dependence on a great variety of parameters (latitude, longitude, solar zenith angle, spectral characteristics of the emission flux, etc.). With current increasing solar activity, such studies become highly challenging. In addition to solving traditional problems of estimating parameters of ionization processes in the ionosphere and problems of reconstructing emission parameters (Mitra, 1974; Liu et al., 1996), the data obtained through the use of GLOBDET can be used to estimate the spatial inhomogeneity of emission fluxes at scales of the Earth's radius.

The high sensitivity of GLOBDET permits us to propose the problem of detecting, in the flare X-ray and EUV ranges, emissions of nonsolar origins which are the result of supernova explosions. 
Acknowledgments. Authors are grateful to A. T. Altyntsev for his encouraging interest in this study and active participations in discussions. Authors are also indebted to O. S. Lesuta, V. V. Grechnev, and K. S. Palamarchouk for preparing the input data. Thanks are also due V. G. Mikhalkovsky for his assistance in preparing the English version of the $\mathrm{T}_{\mathrm{E} X} \mathrm{X}$-manuscript. Finally, the authors wish to thank the referees for valuable suggestions which greatly improved the presentation of this paper. This work was done with support under RFBR grant of leading scientific schools of the Russian Federation No. 00-15-98509 and Russian Foundation for Basic Research (grant 99-05-64753).

\section{References}

Aarons, J., M. Mendillo, and R. Yantosca, GPS phase fluctuations in the equatorial region during sunspot minimum, Radio Sci., 32, 1535-1550, 1997.

Afraimovich, E. L., K. S. Palamartchouk, and N. P. Perevalova, GPS radio interferometry of traveling ionospheric disturbances, J. Atmos. Solar-Terr. Phys., 60, 1205-1223, 1998.

Collin, R. E., Antennas and Radiowave Propagation, McGraw-Hill, New York, 1985.

Davies, K., Ionospheric Radio Waves, Blaisdell Publishing Company, A Division of Ginn and Company, Waltham, Massachusetts-Totonto-London, 1969

Gurtner, W., RINEX: The Receiver Independent Exchange Format Version 2, http://igscb.jpl.nasa.gov/igscb/data/format/ rinex2 . txt, 1993 .

Ho, C. M., B. A. Iijima, X. P. Lindqwister, A. J. Mannucci, L. Sparks, M. J. Reyes, and B. D. Wilson, Ionospheric total electron content perturbations monitored by the GPS global network during two northern hemisphere winter storms, J. Geophys. Res., 103, 26409-26420, 1998.

Klobuchar, J. A., Real-time ionospheric science: The new reality, Radio
Sci., 32, 1943-1952, 1997.

Klobuchar, J. A., Ionospheric time-delay algorithm for single-frequency GPS users, IEEE Transactions on Aerospace and Electronics System, AES 23(3), 325-331, 1986.

Liu, J. Y., C. S. Chiu, and C. H. Lin, The solar flare radiation responsible for sudden frequency deviation and geomagnetic fluctuation, J. Geophys. Res., 101, 10855-10862, 1996.

Mannucci, A. J., C. M. Ho, U. J. Lindqwister, T. F. Runge, B. D. Wilson, and D. N. Yuan, A global mapping technique for GPS-drived ionospheric TEC measurements, Radio Sci., 33, 565-575, 1998.

Mendillo, M., J. A. Klobuchar, R. B. Fritz, A. V. da Rosa, L. Kersley, K. C. Yeh, B. J. Flaherty, S. Rangaswamy, P. E. Schmid, J. V. Evans, J. P. Schodel, D. A. Matsoukas, J. R. Koster, A. R. Webster, and P. Chin, Behavior of the ionospheric $\mathrm{F}$ region during the great solar flare of August 7, 1972, J. Geophys. Res., 79, 665-672, 1974.

Mitra, A. P., Ionospheric Effects of Solar Flares, 294 pp., New Delhi-12, India, 1974.

Pi, X., A. J. Mannucci, U. J. Lindgwister, and C. M. Ho, Monitoring of global ionospheric irregularities using the worldwide GPS network, Geophys. Res. Lett., 24, 2283-2286, 1997.

Saito, A., S. Fukao, and S. Miyazaki, High resolution mapping of TEC perturbations with the GSI GPS network over Japan, Geophys. Res. Lett. 25, 3079-3082, 1998.

Thome, G. D. and L. S. Wagner, Electron density enhancements in the E and $\mathrm{F}$ regions of the ionosphere during solar flares, J. Geophys. Res., 76, 6883-6895, 1971

Vanderveen, K., L. E. Orwig, and E. Tandberg-Hanssen, Temporal correlations between impulsive ultraviolet and hard X-ray busts in solar flares observed with high time resolution, Astrophys. J., 330, 480-492, 1988.

E. L. Afraimovich (e-mail: afra@iszf.irk.ru), E. A. Kosogorov, and L. A. Leonovich 